\title{
Material Form Code
}

National Cancer Institute

\section{Source}

National Cancer Institute. Material Form Code. NCI Thesaurus. Code C93861.

A coded value specifying the state and nature of the material. 